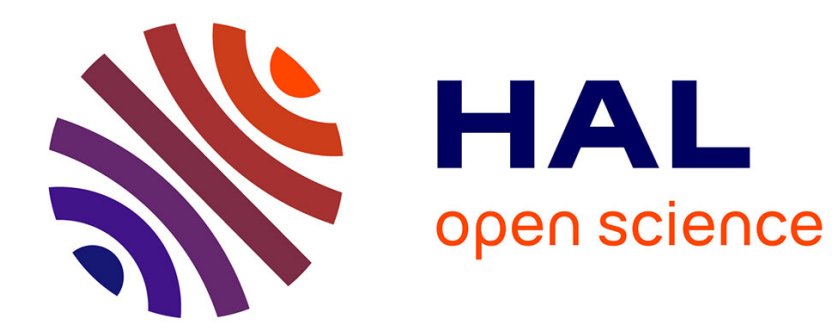

\title{
LE MUSÉE PROTAGONISTE DE L'ART CONTEMPORAIN
}

\author{
Paul Rasse
}

\section{To cite this version:}

Paul Rasse. LE MUSÉE PROTAGONISTE DE L'ART CONTEMPORAIN. Hermès, La Revue -

Cognition, communication, politique, 2011, 61, pp.76-83. hal-03277044

\section{HAL Id: hal-03277044 \\ https://hal.science/hal-03277044}

Submitted on 3 Mar 2022

HAL is a multi-disciplinary open access archive for the deposit and dissemination of scientific research documents, whether they are published or not. The documents may come from teaching and research institutions in France or abroad, or from public or private research centers.
L'archive ouverte pluridisciplinaire HAL, est destinée au dépôt et à la diffusion de documents scientifiques de niveau recherche, publiés ou non, émanant des établissements d'enseignement et de recherche français ou étrangers, des laboratoires publics ou privés. 


\section{LE MUSÉE PROTAGONISTE DE L'ART CONTEMPORAIN}

\section{Paul Rasse}

C.N.R.S. Editions | «Hermès, La Revue »

2011/3 $n^{\circ} 61 \mid$ pages 76 à 83

ISSN 0767-9513

ISBN 9782271072849

Article disponible en ligne à l'adresse :

https://www.cairn.info/revue-hermes-la-revue-2011-3-page-76.htm

Distribution électronique Cairn.info pour C.N.R.S. Editions.

(C) C.N.R.S. Editions. Tous droits réservés pour tous pays.

La reproduction ou représentation de cet article, notamment par photocopie, n'est autorisée que dans les limites des conditions générales d'utilisation du site ou, le cas échéant, des conditions générales de la licence souscrite par votre établissement. Toute autre reproduction ou représentation, en tout ou partie, sous quelque forme et de quelque manière que ce soit, est interdite sauf accord préalable et écrit de l'éditeur, en dehors des cas prévus par la législation en vigueur en France. Il est précisé que son stockage dans une base de données est également interdit. 


\section{Paul Rasse}

Université de Nice Sophia-Antipolis

$I 3 M$

\section{Le musée protagoniste de l'art contemporain}

\begin{abstract}
Depuis sa rénovation, l'institution muséale ne monopolise plus seulement l'attention des experts (les savants, les artistes, les critiques d'art, les érudits) pour lesquels elle avait exclusivement été créée. Elle s'est ouverte sur la société tout entière pour donner à voir, la mise en scène de son patrimoine, c'est-à-dire des traces héritées du passé, enrichies par le présent, destinées à être transmises aux générations futures. Dans la période actuelle où prolifère la création artistique, mais aussi, où s'accélèrent les mutations liées aux progrès de la technoscience, où les réalisations les plus géniales et les plus sophistiquées ont des cycles de vie de plus en plus courts, et deviennent obsolètes avant même d'être usées; au sein de cette immense casse qu'est la postmodernité, les musées sélectionnent les éléments matériels ou immatériels jugés suffisamment significatifs pour mériter d'être conservés et légués comme le témoignage de la culture d'une époque, qu'ils inscrivent dans la continuité historique de celles qui l'ont précédée. Nous défendrons que, dans le domaine de l'art contemporain, les musées jouent un rôle essentiel, sublime, qui confère à l'œuvre la fameuse aura dont Yves Michaud à la suite de Walter Benjamin et l'École de Francfort avaient annoncé la déchéance (Michaud, 1997, p. 65 sqq.;
\end{abstract}

Benjamin, [1935-1939] 2003), alors qu'elle se renouvelle sous de nouvelles configurations ${ }^{1}$.

Quel'on soit pour ou contre les choix des grandes institutions muséales, on peut imaginer sans grand risque de se tromper que les collections d'art contemporain que ces institutions rassemblent et exposent, ou encore, qu'elles installent au milieu des œuvres du passé, constituent et demeureront comme le témoignage esthétique, officiel de la grande culture d'une époque. Dès lors, la problématique est d'ordre épistémologique - au sens où les catégories structurant la connaissance, comme processus d'intelligence collective, sont essentiellement le produit de l'entendement humain. Latour l'a montré pour la science, la vérité est construite: elle se nourrit des théories qui l'ont précédée, de la recherche expérimentale, de controverses, mais surtout elle mobilise des institutions, des réseaux d'acteurs qui vont lui permettre de s'imposer comme une évidence (Latour, 1989). Et cela vaut pour les beaux-arts. Par-delà le débat enflammé auquel se livrent les défenseurs ou les détracteurs de l'art contemporain officiel sur la valeur, l'intérêt ou la beauté des collections, une question essentielle demeure, celle des processus par lesquels l'institution muséale construit 
la vérité esthétique, et par conséquent, celle des processus par lesquels les artistes accèdent à l'espace sacralisant du musée.

\section{Sélectionner, faire circuler, légitimer}

Depuis leurs origines, les musées savent rassembler, discriminer, nommer, organiser, classer, interpréter et donner à savoir; à la différence que, ce qu'ils faisaient pour les savants, ils le font maintenant pour la société entière et notamment dans le domaine de l'art contemporain. Car, après s'y être longtemps refusés, la plupart des conservateurs aspirent à intervenir d'une façon ou d'une autre sur la grande scène de la création contemporaine. Désormais, petits et grands musées s'efforcent d'entrer dans le jeu prestigieux qui vise à distinguer et légitimer le travail des artistes. Ce rôle devient essentiel dans une période qui n'a jamais compté autant d'artistes ${ }^{2}$ et de propositions artistiques, tant les frontières de l'art, du talent ou seulement du professionnalisme sont labiles (Heinich, 1998, p. 75 sqq.); alors que la capacité à produire et à reproduire industriellement des œuvres a bousculé les règles $\mathrm{du}$ jeu et rendu la virtuosité du créateur insignifiante. Plus le travail des artistes transgresse le cadre des tableaux, glisse des cimaises, s'installe dans la nature, gagne les lieux les plus improbables de villes, se dématérialise en images vidéo, se perd ou se construit dans les réseaux digitaux, plus le musée devient l'ultime référent.

L'institution permet aux œuvres sélectionnées d'accéder à l'éternité, car - tout le monde le sait - les collections sont et demeureront incessibles; elles seront, $a d$ vitam aternam, conservées et soigneusement protégées, à l'abri de portes blindées, dans des salles confinées, climatisées. Et même si tout ce qui entre dans le musée n'est pas destiné à être inscrit à l'inventaire, le principe général d'inaliénabilité des œuvres confère une aura à l'ensemble de ce qui y est exposé. Le musée authentifiel'œuvre, il recrée son hic et nunc pour reprendre l'expression de Benjamin, peu importe sa facture, qu'elle ait été démultipliée, copiée, sériée, il la distingue de toutes les autres, l'authentifie et engage le visiteur dans une expérience éthique et sacrée, un rituel de respect et d'adoration (Leveratto, 2006, p. 21 notamment). Ils constituent, incontestablement - par la magnificence et les prouesses architecturales des bâtiments, l'ampleur des moyens mobilisés pour leur financement, le recueillement des foules qui s'y pressent - l'espace sacré de légitimation par lequel certains témoignages de l'art contemporain iront rejoindre la grande chaîne de la culture patrimoniale de l'humanité.

Le musée devient réalisateur d'art contemporain. Il choisit les artistes, met en scène leurs propositions. Les commissaires les rassemblent, les complètent par des prêts d'institution ou des emprunts à des collectionneurs patentés, et les déroulent dans un parcours fluide, surprenant, que les médiateurs s'efforcent de rendre évident pour les visiteurs. Enfin, ils les documentent au travers de catalogues qui feront trace, de dépliants abondamment distribués à l'entrée, et maintenant d'audio-guides, de vidéos et d'applications $i P h o n e$ à télécharger sur le site Internet du musée ${ }^{3}$. Souvent encore, ils y ajoutent des reportages photo ou vidéo sur la genèse de la création, la biographie de l'artiste. Et ils placent tout cela dans la lumière sacralisante de l'institution, sous le regard de cohortes de visiteurs qui se pressent à l'entrée, dans le discours des guides ou des médiateurs à l'intention des touristes, des jeunes scolaires, des étudiants, des publics d'exclus. Et ils la donnent à voir bien au-delà des éclairages de l'exposition, par les médias, les catalogues, les dérivés commercialisés dans les boutiques. La presse, la radio, la télévision reprennent l'événement d'autant plus abondamment que tous les autres en parlent également, que d'immenses placards publicitaires s'en font l'écho sur les murs de villes, et que les boîtes aux lettres postales ou électroniques de tout ce qui compte aux alentours comme 
acteur de la culture, élus politiques, industriels branchés ont reçu l'invitation au vernissage. Et les visiteurs pressés ou patients, amateurs éclairés ou obligés, peuvent en témoigner; eux aussi ont fait l'expérience de la rencontre avec l'œuvre installée dans le sanctuaire, précédée de son halo médiatique. Bref, si les musées la diffusent dans l'imaginaire de la cité, ils sont l'écrin qui abrite l'œuvre authentique et en confirment l'existence. Au final, l'ensemble de ce dispositif est constitutif de l'œuvre, elle ne se réduit plus seulement à la matérialité de la proposition artistique, le musée y ajoute l'aura de l'institution, sa capacité à la légitimer et à la faire rayonner.

\section{Un collège invisible 4}

Aucun musée, si puissant soit-il, n'est en mesure d'imposer seul et à tous ses choix, aucun ne dispose aujourd'hui du pouvoir du prince. Il doit jouer avec les autres. Ensemble ils doivent faire circuler les artistes, estampiller les œuvres. Tous se tiennent, tous reprennent et renforcent le choix des autres, depuis les plus modestes qui peuvent remarquer et faire entrer dans le jeu les jeunes talents, jusqu'aux grandes institutions dont la réputation n'est plus à faire parce qu'ils accueillent depuis toujours les meilleurs et assurent leur consécration dans de grandes rétrospectives, ou mieux encore commandent aux plus célèbres des vivants de grandes expositions monographiques.

La première triennale de Guangzhou, au Guangdong Museum of Art, le Musée d'Art contemporain de Canton, en 2003, nous a donné l'occasion de mettre en évidence quelques-unes des acteurs-clés de ce collège invisible. L'exposition réunissait, pour une rétrospective de l'art contemporain chinois, les plus célèbres plasticiens ayant marqué la décennie précédente. Comme la Chine était restée si longtemps hermétique à toute influence extérieure, il était assez facile de repérer les institutions qui, à partir des années 1980, avaient permis leur reconnaissance. À lire leur biographie, et pour chacun, la liste des grandes expositions qui les ont consacrés (recensées par un imposant catalogue: The First Guangzou Triennal Reinterpretation, 2002), on peut voir scintiller la constellation des villes et des grandes institutions muséales qui ont sélectionné et promu l'art contemporain chinois. Citons: le Contemporary Art Center, et le Queens Museum of Art de New York, le San Francisco Museum of Modern Art, la National Gallery of Canada d'Ottawa, l'Institute of Contemporary Arts à Londres, le Watari Museum of Contemporary Art de Tokyo, et une dizaine, peut-être une quinzaine, d'autres musées célèbres, à Chicago, Vancouver, Sydney, Barcelone, Hanovre, Berlin, Bonn, Bern, auxquels s'ajoutent encore les biennales d'art contemporain de Lyon, de Venise et de Melbourne... Ainsi se dessine une carte mondiale des lieux d'autorité où se forgent les canons esthétiques de l'art contemporain (Rasse, 2006, p. 272-273).

Les musées hiérarchisent les réalisations artistiques en fonction de leur propre position au sein d'une hiérarchie informelle, silencieuse et mondiale, selon qu'ils sont installés à la périphérie ou au centre du système, dans les grandes capitales des pays les plus puissants, ceux du moment ou de leurs prédécesseurs (Quemin, 2001). Ensemble, les grands musées constituent un «collège invisible», un réseau par lequel circulent les artistes reconnus. Ces derniers s'efforçant de consolider leur position et d'accroître leur notoriété, de progresser dans la hiérarchie institutionnelle en se faisant inviter par des institutions toujours plus prestigieuses. Ils y viennent indiscutablement avec un peu de la notoriété des précédents lieux par lesquels ils sont déjà passés, ainsi que le résume et l'égrène leur biographie affichée à l'entrée de l'exposition ou dans les catalogues et les dépliants qui l'accompagnent. Car dans ce jeu, chaque musée doit lui aussi maintenir 
et développer sa réputation, affirmer son rayonnement en choisissant des artistes pour le moins consacrés par d'autres institutions de même niveau, ou mieux encore, en attirant des artistes plus célèbres, estampillés dans des réseaux plus prestigieux, mais en leur offrant, dans ce cas, davantage de place, de moyens et de visibilité. Bien sûr, d'autres éléments entrent en considération, comme le charisme, le talent, les réseaux de relation, l'acharnement, le bon goût, la clairvoyance, les compétences professionnelles des conservateurs et des commissaires d'exposition, mais ces qualités pour essentielles qu'elles soient, jouent à la marge, même si elles font la différence et pencher la balance vers le haut, pour permettre l'ascension de l'institution dans la hiérarchie des musées.

Aucune institution, si prestigieuse soit-elle, n'est à elle seule en mesure d'imposer un artiste, pas plus que ne le sont les conservateurs ou les commissaires d'exposition, mais toutes participent au jeu. Les élus, les bailleurs de fonds, la tutelle attendent d'un musée qu'il invite les meilleurs artistes possibles, c'est-à-dire les plus légitimes, connus pour être passés par d'autres musées au moins aussi réputés, et si possible, des pays les plus puissants du moment. Cependant, sauf exception et à la marge, ils ne peuvent faire mieux que ce que leur autorise leur réputation, les moyens dont ils disposent, leur classement dans la hiérarchie informelle et silencieuse des institutions. De l'autre côté, une carrière bien menée d'artiste impose de s'efforcer de progresser au sein de cette hiérarchie, et en tout cas (même s'il arrive de prêter des œuvres) de concentrer ses efforts et réserve le meilleur de sa création aux institutions les plus célèbres, les plus gratifiantes possibles.

En abîme, les propositions artistiques qui ne réussissent pas à pénétrer le système de légitimation, ou qui y parviennent trop exceptionnellement et par des entrées trop éloignées du centre, sont forcément déclassées, d'autant plus déclassées que ce système hiérarchisé, plus mon- dialisé que jamais, s'étale dans les médias qui s’arrachent les interviews des artistes les plus célèbres et n'ont pas une ligne pour tous les autres.

Bien sûr, il faut encore compter avec le marché (les galeries, les ventes aux enchères) qui représente l'espace où se mesure et s'ajuste la cote des artistes au moyen de l'équivalent général, universel, qu'est leur monétarisation. Il représente aussi la partie la plus lucrative du succès, car les musées achètent les créations à des tarifs bien inférieurs à ceux du marché tout en exigeant des artistes un investissement dans leur projet bien supérieur, à la mesure de la notoriété qu'ils vont, en échange, conférer à leur œuvre tout entière. Comme les grandes institutions financières, les musées assurent et rassurent le marché. Ils constituent un référentiel inaliénable, intemporel, symbolique, comme l'est l'or inaltérable des banques; ils exposent, affichent, placent dans la lumière de grandes matrices esthétiques à partir desquelles les artistes déclinent leurs créations dans des formats moins éphémères et monumentaux, plus appropriés aux attentes et aux intérieurs des collectionneurs. À la sortie du système, les revues d'art, les banques de données accessibles sur le Net publient les cotes, les prix de vente, le classement des meilleurs d'entre eux ${ }^{5}$. Ainsi, par exemple, le Kunstkompass qui fait autorité distingue et classe chaque année les cent premiers artistes en fonction du nombre d'expositions monographiques ou collectives et du nombre d'œuvres achetées par les deux cents plus grands musées du monde, auxquels s'ajoutent encore leur participation aux grandes expositions mondiales et le nombre d'articles qui leur sont réservés dans les magazines d'art, tandis que le prix de vente de leurs réalisations sur les marchés privés ne joue qu'à la marge. À l'autre bout, on trouve encore les grands collectionneurs, qui peuvent contribuer à faire entrer dans le jeu des artistes et des courants moins connus, avant - pour reprendre l'expression de Pomian - qu'«ils ne reçoivent la bénédiction des musées» (Pomian, 2003, p. 350). 


\section{Silence sur le réseau et les procédures d'acquisition}

Jusqu'à une période récente, les conservateurs avaient un savoir taxinomique hérité des musées d'histoire naturelle, ils savaient classer, organiser, discriminer. Ils s'efforçaient de constituer des collections cohérentes, de les regrouper en écoles, en époques, en genres, de les accumuler en nombre pour les constituer en panoptique $\mathrm{du}$ savoir et offrir des ensembles significatifs, nécessaires au progrès de l'art comme de la connaissance à son égard, indispensables à l'inspiration et à la créativité des artistes, à l'enseignement dans les écoles, aux recherches des critiques. Dans tous les cas, les règles étaient évidentes, claires: les musées conservaient et accumulaient des œuvres exceptionnelles, issues des collections royales, des palais princiers, des temples et autres édifices prestigieux, des cathédrales ayant échappé aux outrages du temps et aux avanies de l'histoire, et il n'y en avait pas tant, et c'était bien assez pour leur conférer une valeur exceptionnelle.

Avec l'ouverture des musées à l'art contemporain, le problème a complètement changé d'échelle, d'abord parce qu'il y a profusion de prétendants, d'artistes et d'œuvres, et bien peu de place pour les nouveaux venus dans ce jeu où circulent un peu toujours les mêmes, qui occupent toute la place, ou au moins les meilleures places, celles qui offrent le plus de visibilité et de légitimité à leur œuvre $^{6}$. Dès lors, la question essentielle est celle des choix, qui décide et comment? Ou plus indirectement, comment est-ce qu'on pénètre dans le système institutionnel de légitimation du travail des artistes, et comment est-ce que l'on y progresse?

Le musée dit tout de l'artiste exposé, de sa démarche, de ses références, de son travail, de ce qu'il en dit lui-même et de ce qu'en disent les critiques, mais il ne dit pas, ou si peu d'une question pourtant essentielle, pourquoi eux, pourquoi ce sont eux qui circulent de musées en musées et pas les autres, les milliers d'autres prétendants dont la démarche artistique est probablement parfois aussi innovante, exigeante, cohérente, excentrique, puissante, que sais-je encore pour reprendre les arguments généralement évoqués pour justifier les choix des commissaires d'exposition qui font dépendre leurs décisions des qualités intrinsèques de l'œuvre exposée. Or, nous l'avons vu, les créations ne sauraient se réduire à ce qui est montré d'elles; elles sont constituées de tout le reste, par la démarche et le parcours de l'artiste, par le contexte, le lieu et la lumière de l'exposition, l'aura de l'institution, le regard du public, les catalogues d'exposition, le jeu des médiateurs et de la médiatisation. Les guides d'accompagnement de la visite ne disent pas grand-chose de tout cela, ils apportent bien peu d'informations sur les raisons pour lesquelles certaines œuvres entrent dans la collection. Le système demeure particulièrement opaque ${ }^{7}$.

La fascination qu'exercent l'art contemporain et paradoxalement le rejet dont il fait aussi l'objet de la part des profanes tient sans doute à cela. La colère que suscitent les choix, provient non pas tant des œuvres exposées que des dispositifs de sélection opaques qui les rendent incompréhensibles, irritant pour celui qui voudrait savoir, révoltant pour les prétendants, les artistes qui, sûrs de leur talent, en sont néanmoins exclus. Qui n'a pas entendu ce cri de colère: je ne comprends pas, pourquoi cela ici, alors que moi-même, un enfant, mon voisin artiste amateur à ses heures, pourraient en faire autant! Ce n'est pas tant l'œuvre elle-même qui est mise en question que sa présence, là, dans ce lieu d'exception, extraordinaire, que le choix des commissaires qui ont conduit à l'exposer, elle, et non pas la multitude d'autres propositions possibles.

Le génie des grands artistes ne tient-il pas autant à leurs dons, créatifs, innés, qu'à leurs talents pour se faire reconnaître, qu'à leur capacité à avoir pu pénétrer le système institutionnel pour y exister et faire légitimer leur création. Il est bien difficile d'aller au-delà, de décrypter leur parcours, la part due aux origines sociales, au milieu 
dans lequel ils ont grandi, aux écoles d'art qu'ils ont fréquentées, aux rencontres, aux opportunités qui leur ont été offertes, qu'ils ont suscitées et choisies au bon moment, à leur talent de créateur bien sûr qui a su tout à la fois s'inscrire dans une époque et s'en distinguer, mais aussi aux rencontres, à leurs ambitions, leur ego, leur charisme, leurs sens des relations utiles, leur capacité de séduction, leur intuition, leur intelligence de la communication et bien d'autres qualités encore, conjuguées dans une savante alchimie. Celle-ci leur a permis de pénétrer jusqu'au centre du dispositif, jusqu'au cœur du système de l'art contemporain, mais demeure, heureusement sans doute, indécodable, et déjà parce que les stars de l'art contemporain sont inaccessibles ${ }^{8}$ et se construisent un personnage bien loin de ce qu'ils sont, ou plutôt, de ce qu'ils auraient pu être en d'autres circonstances.

Dans les années 1990, le débat sur l'art contemporain fait rage; à la suite de la crise du marché, philosophes et critiques se sont déchirés et parfois fourvoyés au sujet des contenus, d'un côté la mise en question d'un art

\section{NOTES}

1. Voir l'article de Boris Groys, supra.

2. Ainsi par exemple, en dix ans, le nombre d'artistes plasticiens français affiliés à la maison des artistes a triplé (Gouyon, 2011).

3. Voir, par exemple, les vidéos associées à l'exposition Pop Life. Art in Material World (Tate Modern, $1^{\text {er }}$ oct. 2009 - 17 janv. 2010): Curator Alison Gingeras introduces the exhibition ( 2 min. 59 s.), Damien Hirst: Are You an Identical Twin? (2 min. 13), Jeff Koons' Rabbit in Covent Garden (3 min. 11 s.) ou Opening Party (2 min. 25 s.). En ligne sur <http://www.tate.org.uk/modern/ exhibitions/poplife/explore.shtm>, consulté le 22/08/2011. Ou encore la vidéo Artistic industry in the studio of Jeff Koons (29 janv. 2009, $4 \mathrm{~min} .4 \mathrm{~s}$.). En ligne sur $<$ http://channel.tate.org. uk/media/26522806001>, consulté le 22/08/2011. officiel, déliquescent, convenu dans ses outrances, élitiste, récupéré, de l'autre les défenseurs renvoyant la critique à la réaction, à l'anti-intellectualisme et à la haine fascisantes de tous ceux qui n'ont jamais su reconnaitre les avantgardes et aimer les artistes ${ }^{9}$. Il semble qu'aujourd'hui les passions soient retombées, les œuvres sélectionnées sont dans le temple, elles y demeureront entourées d'une aura qui transcende leur matérialité comme cela existait des reliques sacrées. Quand aux autres, celles des artistes qui n’ont pu pénétrer le temple, elles sont condamnées à disparaître. Il reste encore à faire la critique d'un système de compétition mondialisé, libéral, ultra individualisé auquel tous (les institutions, les artistes, le public...) se soumettent, qui consacre une infime minorité aux dépends de l'immense majorité des autres, dont les moyens pour exister, produire, montrer leur travail vont en se raréfiant, ne serait-ce que parce que le nombre des prétendants est en perpétuelle croissance et que l'attention que leur accorde le public et la société entière va en s'appauvrissant - obnubilée qu'elle est par les lumières de la culture officielle.

4. Pour reprendre une expression utilisée en épistémologie des sciences, quand l'analyse des citations et des références permet d'établir des relations sémantiques entre les articles, de mettre en évidence le collège invisible des chercheurs qui travaillent à distance, sur le même sujet, et de rendre visible le feuilleté des contributions successives par lequel se produit la science, comme une mise en transparence de son architecture.

5. Le Kunstkompass, publié dans le Manager Magazin, est pionnier dans le domaine du classement d'artistes (il a été créé en 1970). Son concurrent, le Kunstmarkt-Kompass, est lui publié dans le magazine Capital, tandis que le portail Artfacts.net diffuse depuis 2003 l'Artist Ranking et, en outre, donne accès à une importante banque de données sur les artistes les plus connus (Friedmann, 2011, p. 222 sqq.). 
6. $50 \%$ des auteurs affiliés à la maison des artistes doivent se partager $15 \%$ de l'ensemble des revenus, tandis que les $10 \%$ d'artistes auteurs les mieux rémunérés concentrent à eux seuls plus de la moitié de ces revenus (Gouyon, 2011).

7. Dans L'Élite artiste (2005), Nathalie Heinich peine à décoder les règles d'un système de recrutement qui pourtant existe, dit-elle, car il permet la démarcation entre grands, moyens et médiocres artiste, si bien qu'au final l'opacité des règles accrédite les représentations d'une indexation de l'excellence sur le privilège de naissance que représente le don inné. De son côté, Pierre-Michel Menger relève lui l'indémêlable imbrication d'individualismes, de réseaux denses, de liens interindividuels et de multiples solutions d'intermédiation... Fiona Friedmann y ajoute encore la capacité à faire du buzz, l'obligation d'entretenir sans cesse des formes d'autopromotion, de communications évènementielles. Et tous de conclure à l'inextricable piège que représente la singu-

\section{RÉ FÉRENCES B I B L I O GR A P H I Q UES}

BARRER, P., Tout l'art contemporain est-il nul?, Lausanne, Favre, 2000 .

BENJAMIn, W., L'Euvre d'art à l'époque de sa reproductibilité technique, Paris, Allia [quatre versions écrites entre 1935 et 1939] 2003.

Clair, J., La Responsabilité de l'artiste, Paris, Gallimard, 1997.

Dagen, Ph., La Haine de l'art, Paris, Grasset, 1997,

DomecQ, J.-P., Misère de l'art. Essai sur le dernier demi-siècle de création, Paris, Calmann-Lévy, [1994] 1999.

FERry, L. et SOllers, Ph., «Débat Ferry/Sollers sur l'art contemporain» in FERry, L., Le Sens du beau. Aux origines de la culture contemporaine, Paris, Cercle d'art, 1998.

The First Guangzhou Triennal. Reinterpretation: A Decade of Experimental Chinese Art, 1990-2000, Catalogue d'exposition, Guangzhou, Guangdong Museum of Art, 2002.

FRIEDMANN, F., Faire carrière sur la scène de l'art contemporain: entre originalité de la création et stratégies d'auto-promotion, Thèse de doctorat, Université de Nice Sophia-Antipolis, 2011. larité de l'artiste, l'obligation de se distinguer, de se démarquer des autres, et en même temps, de se faire reconnaître par l'institution (Heinich, 2005, notamment p. 344 et 349; Menger, 2002, notamment p. 46; Friedmann, 2011).

8. Citons, par exemple, l'artiste Cy Twombly, décédé le 7 juillet 2011. Il occupe la $13^{\mathrm{e}}$ place du Kunstkompass de 2011 et cultivait à l'extrême la discrétion; refusant toute interview, il vivait reclus en Italie dans sa maison de Gaeta, entre Rome et Naples.

9. Citons par exemple: Barrer, 2000; Michaud, 1997; Domecq, [1994] 1999; Dagen, 1997; Ferry, 1998; Clair, 1997. Un certain nombre d'entre eux ont commis l'erreur de publier dans Krisis, une revue obscurantiste suspectée de connivences fascistes, ce qui permit à l'autre clan de vouer les mises en question de l'art contemporain aux dérives d'une idéologie fascisante.

Gouyon, M., «Peintres, graphistes, sculpteurs... Les artistes auteurs affiliés à la Maison des artistes en 2009», Culture chiffres, avril 2011. En ligne sur <http://www2.culture.gouv.fr/culture/ deps/2008/CC-2011-4-MDA-site.pdf>, consulté le 22/08/2011.

HeINICH, N., L'Élite artiste. L'excellence et singularité en régime démocratique, Paris, Gallimard, 2005.

LATOUR, B., La Science en action. Introduction à la sociologie des sciences, Paris, La Découverte, 1989.

Leveratto, J.-M., Introduction à l'anthropologie du spectacle, Paris, La Dispute, 2006.

MARX, K., Le Capital, $4^{\mathrm{e}}$ section du livre $1^{\mathrm{er}}$, t. 2, trad. fr., Paris, Éditions Sociales, [1867] 1973.

Menger, P.-M., Portrait de l'artiste en travailleur. Métamorphoses du capitalisme, Paris, Seuil, 2002.

Michaud, Y., La Crise de l'art contemporain. Utopie, démocratie et comédie, Paris, PUF, 1997.

Pomian, K., Des saintes reliques à l'art moderne, Venise-Chicago, $\mathrm{XIII}^{e}-\mathrm{XX}^{e}$ siècle, Paris, Gallimard, 2003. 
Quemin, A., Le Rôle des pays prescripteurs sur le marché et dans le monde de l'art contemporain, Rapport au ministère des Affaires étrangères, Direction générale de la Coopération internationale et du Développement, juin 2001. En ligne sur <http://www.diplomatie.gouv.fr/fr/IMG/pdf/Le_role_des_pays_prescripteurs_sur_ le_marche_et_dans_le_monde_de_l_art_contemporain.pdf $>$, consulté le 22/08/2011.

RAsse, P., La Rencontre des mondes. Diversité culturelle et communication, Paris, Armand Colin, coll. «Sociétales», 2006. 